\section{Natürliches Interferon hilft gegen hartnäckige schmerzhafte Warzen}

Therapieresistente Patienten profitieren von der Behandlung mit natürlichem Interferon bei Warzen, die durch humanpathogene Papillomaviren ausgelöst werden. Dies zeigt ein aktueller Fall aus Bonn, der im «Journal of Dermatology» beschrieben ist. Bei einem 18-jährigen Patienten mit zahlreichen Warzen an den Zehen zeigten Verätzungen, Kältetherapie sowie Laserbehandlungen keine Wirkung. Erst die intravenöse Gabe von natürlichem Beta-Interferon (Fiblaferon ${ }^{\circledR}$ ) in 3 Zyklen von jeweils 14 Tagen zeigte Erfolg. Einen Monat nach Behandlungsende waren alle Warzen abgeheilt. Nach 12 Monaten war der Patient noch immer beschwerdefrei.

Die Therapie mit natürlichem Beta-Interferon ist besonders bei Patienten sinnvoll, denen herkömmliche Behandlungen nicht helfen oder bei denen wiederholt Infektionen auftreten. Dies war bei dem Bonner Patienten der Fall, dessen zahlreichen Warzen starke Schmerzen verursachten. Durch die antivirale Wirkung begünstigt die Behandlung mit Beta-Interferon nicht nur die schnelle Heilung, sondern verhin- dert außerdem das erneute Auftreten der Viren. Die Erreger siedeln sich auf Haut und Schleimhäuten an und lösen entweder harmlose Warzen oder ernst zu nehmende Virusinfektionen aus. In den meisten Fällen sind die Erkrankungen zwar harmlos, doch in speziellen Fällen können die Warzen starke Schmerzen verursachen.

Weitere Informationen bei biosyn Arzneimittel GmbH, Frau Dr. Gerlinde Reich Tel.: +49 711 57532-17, Fax: -99 E-Mail: Gerlinde_Reich@biosyn.de

\section{Ziele und Forschungsschwerpunkte der Bionorica AG / Bionorica Research GmbH}

Durch die Gründung der Bionorica Research GmbH in Innsbruck soll die weitere Erforschung und Entwicklung von pflanzlichen Wirkstoffen («phyto») in Verbindung mit hochwirksamer Analytik und unter Einsatz neuester Verfahrenstechnologien («engineering») ermöglicht werden. Erklärtes Ziel ist es, innovative pflanzliche Arzneimittel und/oder Arzneimittel auf der Basis von Naturstoffen in kürzestmöglicher Zeit Patienten zur Verfügung zu stellen.

In Innsbruck sollen vor allem die Forschungsschwerpunkte Atemwegsthera- peutika und Medikamente auf Hanf-Basis intensiv verfolgt werden. Im Bereich der pflanzlichen Atemwegstherapeutika sollen Studien zu potenziellen Entwicklungsextrakten durchgeführt werden, die die Datenbasis für eine Entscheidung für den am besten geeigneten «Kandidaten» pflanzlichen Wirkstoff - schaffen sollen. Aber auch Neuentwicklungen von pflanzlichen Arzneien gegen Sinusitis und Allergische Rhinitis sind geplant. Derzeit werden 5 potenzielle Wirkstoffe geprüft. Zusätzlich setzt die Bionorica Research GmbH auf Dronabinol, den Wirkstoff aus der Cannabis-Pflanze. Ein Medikament mit diesem Wirkstoff wäre eine wichtige therapeutische Perspektive und damit ein Hoffnungsträger für viele chronisch erkrankte Patienten. Dronabinol schließt eine therapeutische Lücke für viele derjenigen Patienten, die mit klassischen Arzneimitteln allein nur unzureichend behandelt werden können und leistet einen entscheidenen Beitrag zur Verbesserung ihrer Lebensqualität.

Weitere Informationen bei Bionorica AG, Ingrid Hilfers Tel. +49 9181 231-319

E-mail ingrid.hilfers@bionorica.de

\title{
Ticker+++ Ticker+++ Ticker+++ Ticker+++ Ticker+++ Ticker+++ Ticker+++
}

Pascoe pharmazeutische Präparate GmbH. Von Pascoe Naturmedizin erscheint ein Sonderdruck zum Thema «Vitamin C schützt vor Knochenabbau bei Osteoporose». Vitamin C besitzt einen günstigen Einfluss auf den Knochenmetabolismus, da es essentieller Kofaktor der Kollagen- und Calcitoninsynthese ist. Weitere Informationen und Anforderung des kostenfreien Sonderdrucks bei

Pascoe pharmazeutische Präparate $\mathrm{GmbH}$

Stefanie Wagner-Suske

Tel. +49 641 7960-0, Fax -123

E-mail presse@pascoe.de
Biologische Heilmittel Heel GmbH. Das Komplexhomöopathikum Vertigoheel wird verbreitet bei der Behandlung von Schwindelzuständen unterschiedlicher Genese eingesetzt. Eine neue Studie legt nahe, dass sich die mehrfach belegte Wirksamkeit des Präparats u.a. durch eine verbesserte Sauerstoff- u. Nährstoffversorgung der Zellen und eine erhöhte Anpassungsfähigkeit der Mikrozirkulation an sich ändernde Stoffwechselbedürfnisse erklären lässt. Weitere Informationen bei

Biologische Heilmittel Heel GmbH,

Fachpresseinformation

Tel. +49 7221 501-252, Fax -480

E-mail presse@heel.de
Weleda AG. Am 1. März 2005 wurde gerichtlich entschieden, dass Ärzte ihren Patienten ab sofort uneingeschränkt anthroposophische Mistelpräparate bei der Indikation «maligner Tumor» verordnen können - sowohl adjuvant als auch palliativ. Die Kosten hierfür sind von den gesetzlichen Krankenkassen zu tragen. Weitere Informationen bei

Weleda AG,

Dr. Bettina Arnold-von Versen

Tel. +49 7171 919-325, Fax -447

E-mail barnold@weleda.de 